\title{
Feasibility study for the analysis of coconut water using fluorescence spectroscopy coupled with PARAFAC and SVM methods
}

\begin{abstract}
Purpose: Parallel factor analysis (PARAFAC) coupled with support-vector machine (SVM) was carried out to identify and discriminate between the fluorescence spectroscopies of coconut water brands. Design/methodology/approach: PARAFAC was applied to reduce three-dimensional data of excitation emission matrix (EEM) to two-dimensional data. SVM was applied to discriminate between six commercial coconut water brands in this study. The three largest variation data from fluorescence spectroscopy were extracted using the PARAFAC method as the input data of SVM classifiers. Findings: The discrimination results of the six commercial coconut water brands were achieved by three SVM methods (Ga-SVM, PSO-SVM and Grid-SVM). The best classification accuracies were $100.00 \%, 96.43 \%$ and $94.64 \%$ for the training set, test set and CV accuracy. Originality/value: The above results indicate that fluorescence spectroscopy combined with PARAFAC and SVM methods proved to be a simple and rapid detection method for coconut water and perhaps other beverages.
\end{abstract}

Keyword: Flourecence spectroscopy; Coconut water; PARAFAC algorithm; Rapid detection 